\title{
Níveis da bacitracina de zinco como melhorador do desempenho de frangos de corte
}

\author{
Levels of zinc bacitracin used to improve the performance of broilers
}

\author{
Matheus de Paula Reis ${ }^{I^{*}}$ Paulo Borges Rodrigues ${ }^{\mathrm{II}}$ Vinícius de Souza Cantarelli ${ }^{\mathrm{II}}$ \\ Camila Meneghetti ${ }^{\mathrm{III}}$ Antonio Amandio Pinto Garcia Junior ${ }^{\mathrm{I}}$ Renato Ribeiro de Lima ${ }^{\mathrm{IV}}$ \\ Edison José Fassani ${ }^{I I}$ Luciana de Paula Naves ${ }^{\mathrm{I}}$
}

RESUMO

A utilização de antimicrobianos melhoradores de desempenho na criação de frangos de corte é uma prática comum na indústria avícola e, no Brasil, seu uso deve ser feito de acordo com os limites determinados pelo Ministério da Agricultura Pecuária e Abastecimento (MAPA).Objetivou-se com o presente trabalho determinar o efeito da utilização da bacitracina de zinco, dentro dos níveis recomendados pelo Ministério da Agricultura Pecuária e Abastecimento (MAPA), em dietas para frangos de corte sobre o desempenho de aves e criadas de 1 a 42 dias de idade. Utilizouse o delineamento inteiramente casualizado, com 5 tratamentos, distribuídos em 10 repetições de 25 pintos machos de corte da linhagem Cobb 500 ${ }^{\circledR}$, totalizando 1250 aves. Os tratamentos experimentais foram constituídos de cinco níveis de inclusão de bacitracina de zinco (0, 10, 25, 40 e 55ppm). As variáveis analisadas foram consumo de ração, ganho de peso, conversão alimentar e avaliação econômica das dietas. Para o período total de criação, avaliou-se também a viabilidade dos frangos de corte. A bacitracina de zinco foi eficiente como beneficiador do desempenho de frangos de corte criados no período de 1 a 21, 1 a 35 e 1 a 42 dias. Para o período de 1 a 21 dias, o uso de 55 e 29,3ppm de bacitracina de zinco resultou em menor consumo de ração e maior ganho de peso, respectivamente. No geral, a melhor conversão alimentar foi obtida quando se adicionou 55pm de bacitracina de zinco na ração de frangos de corte.

Palavras-chave: melhorador de desempenho, aditivo zootécnico, antimicrobiano, frango de corte.

\section{ABSTRACT}

The use of growth promoters in the broilers production is a common practice in the poultry industry and its use must be made accordance with limits in Brazil set by the Ministry of Agriculture, Livestock and Supply (MAPA).The main objective of this study was to verify the effect of the use of zinc bacitracin, within the levels recommended by the Brazilian Ministry of Agriculture, Livestock and Food Supply (MAPA), in broiler diets for the performance of birds from 1 to 42 days old. We used a randomized design with five treatments in 10 replications of 25 male chicks from Cobb cut $500^{\circledR}$, totaling 1250 birds. The treatments consisted of five inclusion levels of zinc bacitracin $(0,10$, 25, 40 and 55ppm). The variables studied were feed intake, weight gain, feed conversion and economic evaluation of feed. For the breeding period as a whole, this study also evaluated the viability of broilers. Zinc bacitracin was effective as growth promoter of broilers reared during 1 to 21, 1 to 35 and 1 to 42 days. For the period 1-21 days, using 55 and 29.3ppm of zinc bacitracin resulted in a lower feed intake and increased weight gain, respectively. In general, the best feed conversion was obtained when 55ppm of zinc bacitracin was added to the feed of broilers.

Key words: growth promoter,additive,antimicrobial,broiler.

\section{INTRODUÇÃO}

A produção de frangos de corte passou por fortes transformações nas últimas décadas, obtendo melhora na eficiência de produção. Os grandes avanços do sector avícola, aliados a um clima propício à produção de grãos, utilizados na alimentação das aves, tornou o Brasil o terceiro maior produtor (atrás dos Estados Unidos e da China) e o maior exportador de frangos de corte do mundo. De acordo com o relatório anual 2013 da União Brasileira de Avicultura (UBABEF, 2013), aproximadamente $38 \%$ de toda a carne de frango exportada no mundo

'Programa de Pós-graduação em Zootecnia, Departamento de Zootecnia, Universidade Federal de Lavras (UFLA), 37200-000, Lavras, MG, Brasil. E-mail: matheusreis@zootecnista.com.br.*Autor para correspondência.

IIDepartamento de Zootecnia, UFLA, Lavras, MG, Brasil.

IIIDepartamento de Ciências Agrárias e Ambientais, Universidade Estadual de Santa Cruz (UESC), Ilhéus, BA, Brasil.

${ }^{\text {IV }}$ Departamento de Ciências Exatas, UFLA, Lavras, MG, Brasil. 
em 2012 tiveram origem no Brasil. Essa consolidação de grande produtor de carne de frango é reflexo de um sistema eficaz de nutrição, manejo, genética e ambiente, gerando uma proteína animal de qualidade e acessível à maioria dos consumidores.

MENDES \& SALDANHA (2004) afirmaram que um conjunto de técnicas relacionadas ao manejo, nutrição, vacinas e equipamentos contribuem para fazer da produção avícola brasileira uma atividade econômica com índices de produtividade equivalentes aos observados nos países mais desenvolvidos do mundo. Porém, para a obtenção de alta produtividade, alguns aditivos zootécnicos têm sido usados como melhoradores de desempenho, entre eles, os antimicrobianos melhoradores de desempenho (AMD).

$\mathrm{O}$ uso dos antimicrobianos com o intuito de promover a melhoria no desempenho animal foi proibido em algumas regiões do mundo, principalmente pelos países da Comunidade Europeia (CASEWELL et al.,2003). Essa proibição está relacionada a diversos fatores, dentre eles: seleção de microrganismos resistentes, desenvolvimento de resistência bacteriana cruzada em humanos e à exigência de produtos livres de resíduos de antibióticos pelo mercado consumidor (TRAESEL et al., 2011). Entretanto, por outro lado, estudos indicam que o uso de antimicrobianos para fins terapêuticos aumentaram na Europa, bem como a utilização de anticoccidianos antibióticos (CASTANON, 2007). Outro fato encontrado frequentemente na literatura é a depleção do desempenho animal, associado à proibição dos AMDs (JONES \& RICKE, 2003; AHI, 2006). Segundo a AHI (2009), a proibição dos AMDs na Dinamarca resultou em aumento no aparecimento de doenças, na taxa de mortalide e consequente aumento do uso de antibióticos para fins terapêuticos. A aplicação de forma correta e em níveis adequados pode viabilizar o uso dos antimicrobianos, minimizando os prejuízos e aumentando os lucros do produtor, sem causar riscos à saúde humana.

No Brasil, o uso de antimicrobianos melhoradores de desempenho é permitido e feito dentro de normas estipuladas pelo Ministério da Agricultura Pecuária e Abastecimento (MAPA). Estas normas entraram em vigor a partir de 2004, quando uma das principais mudanças foi a definição de níveis máximos para utilização de cada antimicrobiano, o que levou as empresas que comercializam este aditivo a se adequarem às novas regras. Portanto, existe uma carência por estudos que determinem o melhor nível de utilização dentro dos limites estipulados.
Diante do exposto, objetivou-se com o presente trabalho avaliar o efeito de diferentes níveis, dentro dos limites recomendados pelo MAPA, de bacitracina de zinco, sobre o desempenho e o impacto econômico da inclusão do antimicrobiano na produção de frangos de corte nas diferentes fases de criação.

\section{MATERIAL E MÉTODOS}

O experimento foi conduzido no Departamento de Zootecnia da Universidade Federal de Lavras, localizado na cidade de LavrasMG, no período de outubro a dezembro de 2010. O período experimental foi de 42 dias, no qual foram utilizados 1250 pintos, machos de um dia, da linhagem Cobb-500 ${ }^{\circledR}$, alojados em galpão de alvenaria, sendo distribuídos em 50 boxes. O boxe (parcela/unidade experimental) foi preparado com sistema de aquecimento com lâmpada incandescente de 200watts, bebedouro pendular, comedouro tubular e cama de frango reutilizada, obtida de galpão comercial com o intuito de aumentar a carga microbiológica do ambiente, aproximando o experimento à realidade de campo. A luz foi fornecida durante 24 horas diárias, entre luz natural e artificial, em todo o período de criação.

O delineamento foi inteiramente ao acaso, com cinco tratamentos em 10 repetições cada. Os tratamentos experimentais foram constituídos por cinco níveis de inclusão de bacitracina de zinco $(0,10$, 25, 40 e 55ppm). O antimicrobiano foi incluído à ração em substituição ao material inerte (caulim) da fórmula.

As rações experimentais (Tabela 1) foram formuladas a base de milho e farelo de soja, sendo isonutrientes para cada fase experimental, de acordo com as recomendações nutricionais das Tabelas Brasileiras de Suínos e Aves (ROSTAGNO et al., 2005). Foi adotado o programa alimentar com quatro fases, pré- inicial (1-7 dias), inicial (8-21 dias), crescimento (22-35 dias) e final (36-42 dias). Como fonte do antimicrobiano, utilizou-se a bacitracina de zinco $15 \%$.

As rações foram suplementadas com 90ppm de lasalocida como anticoccidiano, com exceção da fase final de criação, na qual houve a retirada do produto.

Os tratamentos foram sorteados para cada parcela experimental, a ração e a água foram fornecidas ad libitun durante todo o experimento. Os bebedouros foram lavados apenas duas vezes por semana, com o objetivo de aumentar a carga microbiológica do ambiente, aproximando o experimento à realidade de campo. 
Tabela 1 -Composição percentual e níveis nutricionais calculados das rações experimentais (sem antimicrobiano) nas quatro fases de criação.

\begin{tabular}{|c|c|c|c|c|}
\hline \multirow[b]{2}{*}{ Ingredientes } & \multicolumn{4}{|c|}{ 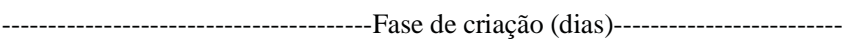 } \\
\hline & 1 a 7 & 8 a 21 & 22 a 35 & 36 a 42 \\
\hline Milho & 53,745 & 57,882 & 60,700 & 64,250 \\
\hline Farelo de soja & 38,850 & 35,330 & 31,800 & 28,164 \\
\hline Óleo de soja & 2,920 & 2,800 & 3,743 & 4,000 \\
\hline Fosfato bicálcico & 1,990 & 1,915 & 1,700 & 1,553 \\
\hline Calcário calcítico & 0,893 & 0,805 & 0,810 & 0,769 \\
\hline Sal comum iodatado & 0,516 & 0,493 & 0,450 & 0,443 \\
\hline Suplemento mineral ${ }^{1}$ & 0,100 & 0,100 & 0,100 & 0,120 \\
\hline Suplemento vitamínico ${ }^{2}$ & 0,100 & 0,100 & 0,100 & 0,120 \\
\hline DL-metionina 99\% & 0,340 & 0,235 & 0,250 & 0,220 \\
\hline L-lisina HCL 78\% & 0,284 & 0,155 & 0,170 & 0,203 \\
\hline L-treonina 98\% & 0,112 & 0,035 & 0,027 & 0,047 \\
\hline Cloreto de colina 60\% & 0,050 & 0,050 & 0,040 & 0,020 \\
\hline Lasalocida 15\% & 0,060 & 0,060 & 0,060 & 0,000 \\
\hline Caulim & 0,040 & 0,040 & 0,050 & 0,090 \\
\hline Total & 100,000 & 100,000 & 100,000 & 100,000 \\
\hline Energia metabolizável (kcal/kg) & 2950 & 3000 & 3100 & 3150 \\
\hline Proteína bruta (\%) & 22,04 & 20,79 & 19,41 & 18,03 \\
\hline Lisina digestível (\%) & 1,33 & 1,146 & 1,073 & 1,017 \\
\hline Metionina+cistina digestível (\%) & 0,944 & 0,814 & 0,773 & 0,732 \\
\hline Treonina digestível (\%) & 0,865 & 0,745 & 0,697 & 0,661 \\
\hline Cálcio (\%) & 0,939 & 0,884 & 0,824 & 0,763 \\
\hline Fósforo disponível (\%) & 0,47 & 0,442 & 0,411 & 0,38 \\
\hline Sódio (\%) & 0,223 & 0,214 & 0,205 & 0,194 \\
\hline
\end{tabular}

${ }^{1}$ Enriquecimento por kg de ração: Vitamina A - 12.000UI, Vit.D - 2.200UI, Vit E - 30mg, Vit K - 2,5mg, Niacina - 53mg, Ácido fólico 1,0mg, Ácido pantotênico - 13mg, Biotina - 110mg, Vit B1 - 2,2mg, Vit B2 - 6mg, Vit B6 - 3,3mg, Vit B12 - $16 \mu$ g e Selênio - 0,25 mg.

${ }^{2}$ Enriquecimento por kg de ração: Ferro - 50mg, Cobre - 8,5mg, Zinco - 70mg, Manganês -75mg, Iodo - 1,5mg e Cobalto - 0,2 mg.

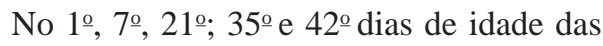
aves, as sobras de ração e as aves foram pesadas para avaliação do consumo de ração, ganho de peso e conversão alimentar nos períodos correspondentes a: 1 a $7 ; 1$ a 21; 1 a 35 e 1 a 42 dias de idade. Por meio do monitoramento diário da mortalidade das aves, efetuou-se o estudo da viabilidade, compreendendo o período total de criação.

Realizou-se também um estudo econômico das dietas utilizadas. Foi utilizada uma metodologia adaptada de SCHEUERMANN(2009) e GOMES (2012), descrita pela fórmula abaixo:

Avaliação econômica $(\mathrm{R} \$ / \mathrm{kg})$ = consumo de ração $(\mathrm{kg}) \times$ custo da dieta $\left(\mathrm{R} \$ \mathrm{~kg} \mathbf{- 1}^{-1}\right)$. Ganho de peso(kg)

Os valores utilizados para a composição do custo das dietas foram obtidos na região de Lavras - MG, no mês de junho de 2013, sendo: milho $(\mathrm{R} \$ 0,46)$, farelo de soja $(\mathrm{R} \$ 1,10)$, óleo de soja refinado ( $\mathrm{R} \$ 2,80)$, fosfato bicálcico $(\mathrm{R} \$ 1,20)$, calcário calcítico ( $\mathrm{R} \$ 0,70)$, sal comum $(\mathrm{R} \$ 0,42)$, suplemento vitamínico (R\$6,81), suplemento mineral (R\$4,86), DL- metionina (R\$ 13,90), L-lisina $\mathrm{HCl}$
(R\$7,00), L-treonina ( $\mathrm{R} \$ 8,90)$, cloreto de colina $60 \%$ $(\mathrm{R} \$ 4,90)$, lasalocida $(\mathrm{R} \$ 6,85)$ e bacitracina de zinco $15 \%(\mathrm{R} \$ 11,50)$.

Os dados foram submetidos à análise de regressão pela decomposição polinomial dos graus de liberdade, referentes aos níveis de bacitracina de zinco. Para a análise dos dados, foi utilizado o software Sistema de Análise de Variância para dados balanceados (SISVAR), descrito por FERREIRA (2010).

Os dados foram analisados, segundo o modelo estatístico:

$$
Y i j=b 0+b 1 i+b 2 i+e i j
$$

em que:

Yij: observação da variável dependente na unidade experimental j submetida ao nível i de Bacitracina de zinco, i: 1 , 2, 3, 5 (1=0ppm; 2=10ppm; 3=25ppm; 4=40ppm e $5=55 \mathrm{ppm})$;

b0: constante;

b1 e b2: são, respectivamente, coeficientes linear e quadrático de regressão da variável dependente em função dos níveis de Bacitracina de zinco; eij: erro aleatório associado a cada observação Yij. 


\section{RESULTADOS E DISCUSSÃO}

O resultado de desempenho das aves pode ser visualizado na tabela 2.

No período de 1a 7dias de idade, os resultados de desempenho (consumo de ração CR, ganho de peso - GP e conversão alimentar - CA) mostraram não haver efeito $(\mathrm{P}>0,05)$ dos níveis de bacitracina na ração sobre nenhuma das características avaliadas. Este resultado já era esperado, considerando a baixa concentração de microrganismos durante a fase pré-inicial, pois, segundo DIBNER \& RICHARDS (2005), os animais nascem isentos de microbiota intestinal e começam a adquiri-la exatamente após a eclosão. Portanto, a importância do uso dos AMDs na fase pré-inicial pode estar relacionada à seleção de microrganismos menos agressivos ao hospedeiro, porém, o efeito sobre o desempenho das aves começa a ser observado após a fase pré-inicial de criação.

Tabela 2 -Consumo de ração (CR), ganho de peso (GP), conversão alimentar (CA), análise econômica (AE) e viabilidade (Viabil.) dos frangos de corte, nas fases de 1 a 7; 1 a 21; 1 a 35; 1 a 42 dias, submetidos às dietas contendo diferentes níveis de bacitracina de zinco.

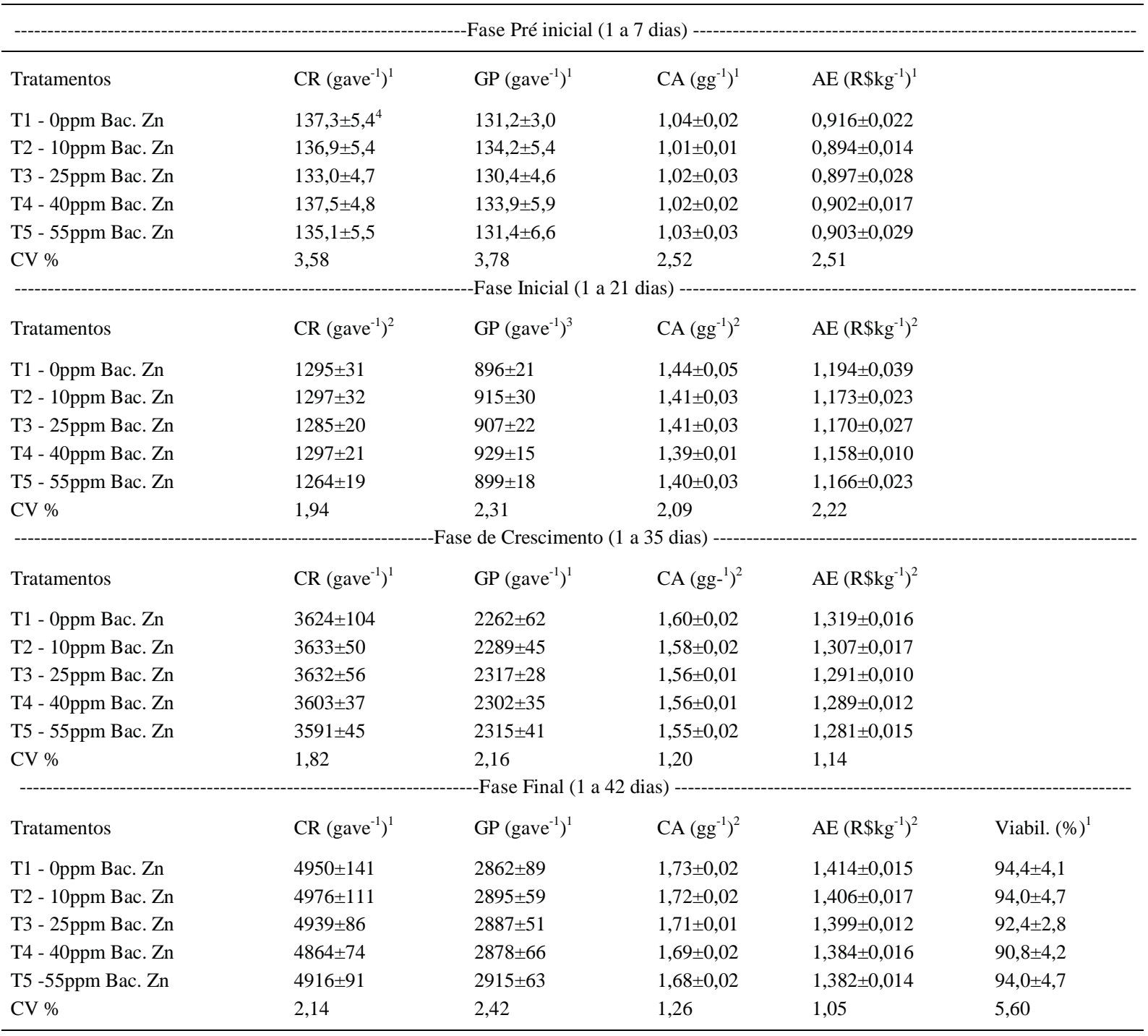

\footnotetext{
${ }^{1}$ Médias não diferem estatisticamente pelo teste $\mathrm{F}$ ao nível de $5 \%$ de probabilidade.

${ }^{2}$ Efeito linear $(\mathrm{P}<0,05)$.

${ }^{3}$ Efeito quadrático $(\mathrm{P}<0,05)$.

${ }^{4}$ Desvio padrão da média.
} 
Avaliando o desempenho das aves no período de 1a 21 dias, foi observado efeito significativo dos níveis de bacitracina de zinco na ração para todas as variáveis estudadas $(\mathrm{P}<0,05)$. Para a variável consumo de ração, observou-se resposta linear decrescente (CR gave $^{-1}=-0,458( \pm 0,180)$ $\left.\mathrm{x}+1299,562( \pm 5,901) ; \quad \mathrm{R}^{2}=50,42 \%\right)$, com menor consumo (1274gave ${ }^{-1}$ ) quando se utilizou 55ppm de bacitracina de zinco. Analisando os níveis de bacitracina na ração para o ganho de peso, observouse resposta quadrática (GP gave ${ }^{-1}=-0,027( \pm 0,009)$ $\left.\mathrm{x}^{2}+1,568( \pm 0,568) \mathrm{x}+897,036( \pm 6,186) ; \quad \mathrm{R}^{2}=52,84 \%\right)$, com estimativa de inclusão de 29,037ppm para máximo ganho de peso. Por meio da análise de regressão, foi possível observar que, apesar da equação estimar maior consumo de ração para as aves que não receberam antimicrobiano, o maior consumo não foi suficiente para incrementar o ganho de peso. Estes resultados estão de acordo com outros estudos (ENGBERG et al., 2000; BRENNAN et al., 2003), nos quais houve melhora no ganho de peso das aves, justificado pela diferença no perfil microbiológico do intestino.

Observou-se efeito positivo do uso de bacitracina de zinco na ração sobre a conversão alimentar das aves, com resposta linear dos níveis (CA gg $^{-1}=-0,000675( \pm 0,000225) \mathrm{x}+1,434( \pm 0,007)$; R2=69,73\%), estimando melhor conversão $\left(1,396 g^{-1}\right)$ quando se utilizou 55pm de bacitracina na ração. A pior conversão $\left(1,445 g^{-1}\right)$ verificada para as aves que não receberam bacitracina na ração, representou uma diferença de aproximadamente 3,4\% na conversão alimentar, com relação às aves que receberam a bacitracina de zinco. Foi possível observar que as aves suplementadas com bacitracina de zinco reduziram o consumo de ração, entretanto, esse fato não foi acompanhado por redução no ganho de peso, demonstrando maior eficiência em aproveitar os nutrientes da dieta, melhorando a conversão alimentar.

Pelos resultados do desempenho das aves no período de 1a 35 dias de idade, nota-se que, diferentemente dos resultados obtidos de 1 a 21 dias, não houve diferença significativa $(\mathrm{P}>0,05)$ para $\mathrm{o}$ consumo de ração e ganho de peso. O mesmo resultado foi observado por BRENNAN et al. (2003), quando compararam o ganho de peso de aves que receberam bacitracina em relação ao tratamento controle (sem o antimicrobiano), e não verificaram diferença significativa $(\mathrm{P}>0,05)$ para o consumo de ração, no período de 1 a 35 dias de idade das aves. Diferente do observado para o consumo de ração e para o ganho de peso, verificou-se melhor conversão alimentar para os tratamentos que receberam bacitracina de zinco (CA $\mathrm{gg}^{-1}=-0,000855( \pm 0,000121) \mathrm{x}+1,596819( \pm 0,003989)$;
$\mathrm{R}^{2}=93,53 \%$ ), obtendo-se o menor valor quando se utilizou o nível de 55ppm, representando melhora de $3,2 \%$ na conversão, em relação ao tratamento com 0ppm de bacitracina.

Ao observar o desempenho das aves no período total de criação (1 a 42 dias de idade das aves), similar ao ocorrido na fase anterior, não foi observado resultado significativo $(\mathrm{P}>0,05)$ para o consumo de ração e para o ganho de peso. Entretanto, notou-se que a conversão alimentar das aves que receberam os diferentes níveis de bacitracina na ração, da mesma forma que o observado nos períodos de 1 a 21 e 1 a 35 dias, apresentou efeito linear $(\mathrm{P}<0,001) \quad\left(\mathrm{CA}\right.$ gg $^{-1}=-0,000820( \pm 0,000129)$ $\left.\mathrm{x}+1,728551( \pm 0,004235) ; \mathrm{R}^{2}=96,22 \%\right)$, com menor conversão alimentar $\left(1,68 g^{-1}\right)$ na suplementação de $55 \mathrm{ppm}$ de bacitracina, representando uma melhora de $2,5 \%$ em relação às aves que não receberam a bacitracina (0ppm). Apesar de não haver diferença significativa no consumo de ração e no ganho de peso para este período, na fase inicial (1 a 21), verificaramse diferenças estatísticas para estas variáveis, o que pode ter sido responsável pela melhora na conversão alimentar no período total, demonstrando a importância da aplicação de bacitracina de zinco nos períodos iniciais de criação. VIOLA \& VIEIRA (2007) observaram benefícios sobre a conversão alimentar na ausência de alterações no ganho de peso, como obtido neste estudo. Segundo os autores, esse fato denota a melhora na utilização de nutrientes a partir de uma mesma quantidade de alimento consumida, supondo que tenha havido redução em desafios microbiológicos pela ação dos antimicrobianos.

Para a variável viabilidade de criação, não foram encontradas diferenças significativas $(\mathrm{P}>0,05)$ para os níveis de bacitracina avaliados, demostrando que este índice foi pouco influenciado pela adição de antimicrobiano na dieta.

Com relação à análise econômica, foi possível observar diferença significativa $(\mathrm{P}<0,05)$ para as três últimas fases de criação (1 a 21, 1 a 35 e 1 a 42 dias), nas quais os níveis de bacitracina utilizados, apresentaram

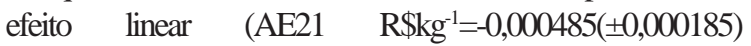
$\mathrm{x}+1,184817( \pm 0,006062), \quad \mathrm{R}^{2}=64,36 \% ; \quad$ AE35 $\mathrm{R} \$ \mathrm{~kg}^{-1}=$ $0,000661( \pm 0,000104) x+1,314584( \pm 0,003434)$, $\mathrm{R}^{2}=91,62 \% ; \quad \mathrm{AE} 42 \quad \mathrm{R} \$ / \mathrm{kg}^{-1}=-0,000612( \pm 0,000104)$ $\left.x+1,412904( \pm 0,003434), \quad R^{2}=95,97 \%\right)$. Os resultados dessavariáveldemonstram queaadiçãodoantimicrobiano, reduziu o custo gasto com alimentação, por quilo de peso vivo produzido, em aproximadamente $1 \%$, ou seja, foi possível produzir a mesma quantidade ou mais de carne, com menor quantidade de ração. 
Essa variável reforça os resultados observados para as variáveis de desempenho, nas quais a conversão alimentar também foi melhorada com a adição do produto nas mesmas fases de criação.

\section{CONCLUSÃO}

As aves apresentaram melhora no ganho de peso e consumo de ração somente na fase de 1 a 21 dias, ao suplementar 29,3 e 55ppm de bacitracina de zinco à ração, respectivamente. Houve melhora na conversão alimentar e na análise econômica, nos períodos de 1 a 21, 1 a 35 e 1 a 42 dias, ao adicionar $55 p p m$ de bacitracina de zinco à ração das aves, porém o consumo de ração e o ganho de peso não apresentaram efeito significativo no período total de criação, demonstrando que o antimicrobiano testado foi eficiente em melhorar o desempenho das aves até os 21 dias e apenas a conversão alimentar e a análise econômica nos períodos posteriores.

\section{COMITÊ DE ÉTICA E BIOSSEGURANÇA}

O protocolo de experimentação animal no048/2009.

\section{AGRADECIMENTOS}

Ao Conselho Nacional de Desenvolvimento Científico e Tecnológico (CNPq) (Processo número - 373716/2010-9) e à Coordenação de Aperfeiçoamento de Pessoal de Nível Superior (Capes) pelo fornecimento de recursos e bolsas, contribuindo de maneira essencial para o desenvolvimento da pesquisa.

\section{REFERÊNCIAS}

AHI(ANIMAL HEALTH INSTITUTE). Political bans on antibiotics are Counterproductive European test case: increased animal disease, mixed human health benefit, 2006. Disponível em: <http://www.ahi.org/issues-advocacy/animal-antibiotics/>. Acesso em: 15 jul. 2011.

ALP, M. et al. Effects of dietary supplementation with organic acids and zinc bacitracin on ileal microflora, $\mathrm{pH}$ and performance in broilers. Turkish Journal of Veterinary and Animal Sciences, Ankara, v.23, n.5 p.451-455, 1999. Disponível em: <http:// journals.tubitak.gov.tr/veterinary/issues/vet-99-23-5/vet-23-5-898069.pdf>. Acesso em: 06 jul. 2011.

BAURHOO, B. et al. Effects of diets containing different concentrations of mannanoligosaccharide or antibiotics on growth performance, intestinal development, cecal and litter microbial populations, and carcass parameters of broilers. Poultry Science, Champaign, v.88, n.11, p.2262-2272, 2009. Disponível em: <http:// ps.fass.org/content/88/11/2262.full.pdf>. Acesso em: 06 jul. 2011. doi: 10.3382/ps.2008-00562.

BRENNAN, J. et al. The efficacy of bacitracin methylene disalicylate when fed in combination with narasin in the management of necrotic enteritis in broiler chickens. Poultry
Science, Champaign, v.82, n.3, p.360-363, 2003. Disponível em: $<$ http://ps.fass.org/content/82/3/360.full.pdf>. Acesso em: 05 jun. 2011.

CASEWELL, M. et al. The European ban on growth-promoting antibiotics and emerging consequences for human and animal health. Journal of Antimicrobial Chemotherapy, Cambridge, v.52, n.2, p.159-161, 2003. Disponível em: <http://jac. oxfordjournals.org/content/52/2/159.full.pdf >. Acesso em: 26 jul. 2011. doi: $10.1093 / \mathrm{jac} / \mathrm{dkg} 313$.

CASTANON, J. History of the use of antibiotic as growth promoters in European poultry feeds. Poultry Science, Champaign, v.86, n.11, p.2466-2471, 2007.Disponível em: <http:// ps.fass.org/content/86/11/2466.full.pdf>. Acesso em: 20 jul. 2011. doi: 10.3382/ps.2007-00249.

DIBNER, J.; RICHARDS, J. Antibiotic growth promoters in agriculture: History and mode of action. Poultry Science, Champaign, v.84, n.4, p.634-643, 2005. Disponível em: <http:// ps.fass.org/content/84/4/634.full.pdf>. Acesso em: 06 jul. 2011

ENGBERG, R.M. et al.Effect of zinc bacitracin and salinomycin on intestinal microflora and performance of broilers. Poultry Science, Champaign, v.79, n.9, p.1311-1319, 2000.

FERREIRA, D.F. Sistema para análise de variância para dados balanceados - SISVAR 5.3. Lavras: UFLA, 2010. Software. Disponível em: <http://ps.fass.org/content/79/9/1311.full.pdf> . Acesso em: 07 jul. 2011

GOMES, T.R. et al. Efeito da inclusão de farelo de arroz integral em rações para leitões de 21 a 42 dias de idade. Archivos de Zootecnia, Córdoba, v.61, n.233, p.129-139, 2012. Disponível em:<http://www.uco.es/organiza/servicios/publica/az/php/img/ web/13_13_18_14-2173FareloGomes.pdf>. Acesso em: 25 jun. 2013. doi: 10.1590/S0103-84782009000200032.

JONES, F.; RICKE, S. Observations on the history of the development of antimicrobials and their use in poultry feeds. Poultry Science, Champaign, v.82, n.4, p.613-617, 2003. Disponível em: <http:// ps.fass.org/content/82/4/613.full.pdf>. Acesso em: 30 jul. 2011.

MENDES, A.A.; SALDANHA, É.S.P.B. Produção de frango de corte. A cadeia produtiva da carne de aves no Brasil. Campinas:Facta. 2004. 356p.

MEURER, R.F.P. et al.Evaluation of the use of probiotics in diets with or without growth promoters for broiler chicks. Revista Brasileira de Zootecnia, Viçosa, v.39, n.12, p.2687-2690, 2010. Disponível em:<http://www.scielo.br/pdf/rbz/v39n12/a19v39n12. pdf $>$. Acesso em: 21 jul. 2011.

ROSTAGNO, H.S. et al. Tabelas brasileiras para aves e suínos: composição de alimentos e exigências nutricionais.Viçosa: UFV, 2005. 186p

SCHEUERMANN, G.N. et al. Phytogenic additive as an alternative to growth promoters in broiler chickens. Ciência Rural, Santa Maria, v.39, n.2, p.522-527, 2009. Disponível em:<http://www. scielo.br/pdf/cr/v39n2/a32v39n2.pdf>. Acesso em: 25 jun. 2013. doi: 10.1590/S0103-84782009000200032.

SILVA, M.A. et al. Óleo essencial de aroeira-vermelha como aditivo na ração de frangos de corte. Ciência Rural, Santa Maria, v.41, n.4, p.676-681, 2011. Disponível em: <http://www.scielo. 
br/pdf/cr/v41n4/a923cr3695.pdf>. Acesso em: 20 dez. 2011. doi: 10.1590/S0103-84782011005000034

SIMS, M.D. et al. Effects of dietary mannan oligosaccharide, bacitracin methylene disalicylate, or both on the live performance and intestinal microbiology of turkeys. Poultry Science, Champaign, v.83, n.7, p.1148-1154, 2004. Disponível em: <http:// ps.fass.org/content/83/7/1148.full.pdf>. Acesso em: 20 jul. 2011.

TRAESEL, C.K. et al. Óleos essenciais como substituintes de antibióticos promotores de crescimento em frangos de corte: perfil de soroproteínas e peroxidação lipídica. Ciência Rural, Santa Maria, v.41, n.2, p.278-284, 2011. Disponível em: <http://www. scielo.br/pdf/cr/v41n2/a867cr3715.pdf>. Acesso em: 13 out. 2011. doi: 10.1590/S0103-84782011000200016.

UBABEF (UNIÃO BRASILEIRA DE AVICULTURA). Relatório Anual 2013. Disponível em: <http://www.abef.com. br/files/publicacoes/723e684103de4a2117dda9ddd280a.pdf >. Acesso em: 25 jul. 2013.

VIOLA, E.S.; VIEIRA, S.L. Suplementação de acidificantes orgânicos e inorgânicos em dietas para frangos de corte: desempenho zootécnico e morfologia intestinal. Revista Brasileira de Zootecnia, Viçosa, v.36, n.7, p.1097-1104, 2007. Disponível em: <http://www. scielo.br/pdf/rbz/v36n4s0/16.pdf>. Acesso em: 15 jul. 2011. 